\title{
MiR-200a-3p promoted the malignant behaviors of ovarian cancer cells through regulating PCDH9
}

This article was published in the following Dove Press journal: OncoTargets and Therapy

\section{Can Shi \\ Yijun Yang \\ Lei Zhang \\ Juanpeng Yu \\ Shanshan Qin \\ Hongge $\mathrm{Xu}$ \\ Yingchun Gao}

Department of Obstetrics and Gynecology, The Affiliated Huaian No. I People's Hospital of Nanjing Medical University, Huai'an, Jiangsu 223300 ,

People's Republic of China
Correspondence: Yingchun Gao Department of Obstetrics and Gynecology, The Affiliated Huaian No. I People's Hospital of Nanjing Medical University, \#I Huanghe Road West, Huai'an, Jiangsu 223300, People's Republic of China

Email gaocail40407@I26.com
Background: Increasing evidence has revealed that the aberrant expression of microRNAs (miRNAs) plays vital roles in the development and progression of ovarian cancer. MiR-200a$3 p$ was found to act as an oncogene in a variety of cancers, however, the expression and function of miR-200a-3p in ovarian cancer has not been characterized.

Materials and methods: The expression of miR-200a-3p in ovarian cancer tissues and cell lines was detected by the RT-qPCR. The influence of miR-200a-3p on the growth of ovarian cancer cells was determined with the Cell Counting Kit- 8 assay, colony formation and cell invasion assay. The binding of miR-200a-3p with the 3'-untranslated region (UTR) of PDCH9 was detected by luciferase reporter assay. The expression of PCDH9 was investigated by RT-qPCR and Western blot analysis.

Results: miR-200a-3p was up-regulated in ovarian cancer tissues and cell lines. Highly expressed miR-200a-3p was significantly associated with the tumor size, tumor metastasis and TNM stage. Overexpression of miR-200a-3p markedly promoted the proliferation, colony formation and invasion of ovarian cancer cells. Functional study uncovered that miR-200a-3p bound the 3'-untranslated region (UTR) of PCDH9 and decreased the expression of PCDH9 in ovarian cancer cells. The expression of miR-200a-3p in ovarian cancer tissues was significantly negatively correlated with that of PCDH9. Restored PCDH9 inhibited the promoting effect of miR-200a-3p on the proliferation of ovarian cancer cells. Conclusion: Our results suggested the potential oncogenic function of miR-200a-3p via modulating PCDH9 in ovarian cancer.

Keywords: miR-200a-3p, ovarian cancer, PCDH9

\section{Introduction}

Ovarian cancer (OC) is the most lethal malignant gynecological cancer with the majority of patients diagnosed at the advanced stage. ${ }^{1-3}$ Despite the remarkable progress in the treatment of OC including surgical resection, chemotherapy and radiotherapy, the 5-year overall survival rate of the OC patients remains less than $40 \%{ }^{4}$ OC has a high recurrence rate and tends to develop the resistance to the current therapy strategies. Therefore, it is vital to explore the molecular mechanisms involved in the progression of $\mathrm{OC}$ and identify novel targets with prognostic and therapeutic significance.

MicroRNAs (miRNAs) are a class of single stranded non-coding RNAs that down-regulate the expression of target genes via binding the 3 '-untranslated regions (3'-UTR). ${ }^{5,6}$ The binding of miRNA results in the degradation or translation inhibition of targeted mRNAs. ${ }^{7,8}$ Dysfunction of miRNAs is frequently associated with a variety of biological processes, including cell proliferation, differentiation and 
apoptosis. ${ }^{9}$ Recent studies demonstrated that the dysregulation of miRNAs significantly contributes to the development and progression of ovarian cancer. ${ }^{10-12}$ For example, Zhou et al found that miR-183 modulated the proliferation and apoptosis of ovarian cancer cells via targeting the TGF$\beta /$ Smad4 pathway. ${ }^{13}$ Overexpression of miR-598 inhibited the growth and metastasis of ovarian cancer cells by regulating the expression of URI. ${ }^{14}$ MiR-34c targeted SOX9 and regulated the sensitivity of ovarian cancer cells to cisplatin-based chemotherapy. ${ }^{15}$ Interestingly, the potential oncogenic function of miR-200a-3p in cancers was recently reported, which indicated the prognosis and therapeutic importance of miR-200a-3p in cancer. ${ }^{16-18}$ However, the role of miR-200a-3p in ovarian cancer remains largely unknown.

The cadherin superfamily is a large group of transmembrane or membrane-associated glycoprotein, which plays important roles in the embryogenesis and maintaining the normal tissue structure. ${ }^{19,20}$ Some cadherins, such as E-cadherin, were reported as tumor suppressor genes (TSG) in the invasion and metastasis of cancer cells. ${ }^{20}$ The protocadherins (PCDHs) belonging to the cadherin superfamily are a group of calcium-dependent adhesion proteins. ${ }^{21,22}$ Recent studies showed that PCDH8, PCDH10 and PCDH17 acted as tumor suppressors in a variety of human cancers. ${ }^{23-34}$ PCDH9 is another member of the PCDH family located in the $13 \mathrm{q} 21.32$ of human genome. Previous studies demonstrated that PCDH9 was down-regulated in cancers, such as glioblastoma, hepatocellular carcinoma and gastric cancer. ${ }^{35-38}$ Overexpression of PCDH9 inhibited the proliferation, migration and invasion of cancer cells. However, the function of PCDH9 in ovarian cancer remains largely unknown.

The aim of this study was to explore the role of miR$200 a-3 p$ in the progression of ovarian cancer. It was observed that miR-200a-3p was up-regulated in ovarian cancer tissues and cell lines. Functional studies uncovered that miR-200a-3p inhibited the proliferation of ovarian cancer cells via targeting PCDH9. These findings revealed the functional mechanism of miR-200a-3p in regulating the growth of ovarian cancer cells and shed light on the clinical significance of miR-200a-3p/PCDH9 axis in ovarian cancer.

\section{Materials and methods}

\section{Tissue samples}

A total of fifty paired ovarian cancer tissues and adjacent normal tissues (at least $5 \mathrm{~cm}$ from primary tumor margin) were obtained from the ovarian cancer patients via surgical resection at The Affiliated Huai'an No. 1 People's Hospital of Nanjing Medical University from August 2010 to December 2013. The tissues were immediately frozen in liquid nitrogen and stored at $-80{ }^{\circ} \mathrm{C}$ prior to the experiments. Written informed consents were obtained from all the patients. This study was approved by the Ethics Committee of The Affiliated Huai'an No. 1 People's Hospital of Nanjing Medical University.

\section{Cell culture and transfection}

Ovarian cancer cell lines including ES2, HO8919PM, SKOV3, HO8910 and the ovarian surface epithelial cells HOSEpiC were purchased from the Chinese Academy of Sciences (Shanghai, China). HO8919PM and HO8910 cells were cultured in RPMI1640 medium containing $10 \%$ fetal bovine serum (FBS, Corning, NY, USA) and $1 \%$ streptomycin/penicillin at $37{ }^{\circ} \mathrm{C}$ with $5 \% \mathrm{CO}_{2}$. SKOV3 and ES2 were cultured with the McCoy's 5A modified medium supplemented with $10 \%$ FBS. The cell transfection was performed with the Lipofectamine 2000 (Invitrogen) according to the manufacturer's instructions.

\section{RNA extraction and quantitative PCR}

Total RNA was extracted from tissues or cell lines using the Trizol reagent (Invitrogen) according to the manufacturer's protocol. The isolated RNA was reverse transcripted into cDNA using the Revert Aid First Stand cDNA synthesis kit (Thermo Fishers Inc., USA) with random primers. The expression of miR-200a-3p was determined by quantitative real-time PCR with the SYBR master mix (Takara, Dalian, China) in according with the manufacturer's instructions. The experiment was performed in triplicate and the expression of U6 RNA was detected as the internal control. The relative expression of miR-200a-3p was calculated with the $2^{-\Delta \Delta \mathrm{Cq}}$ method. The primers used in this study were designed as follows: miR200a-3p forward: 5'-TAACACTGTCTGGTAACGATGT and reverse: 5'-CATCTTACCGGACAGTGCTGGA; U6 forward: 5'- CTCGCTTCGGCAGCACA and reverse: 5'AACGCTTCACGAATTTGCGT. The primers were synthesized in Sangon Biotech Inc. (Shanghai, China).

\section{Cell proliferation assay}

The proliferation of ovarian cancer cells transfected with miR-200a-3p mimics or scramble control miRNA was determined by the Cell Counting Kit-8 (CCK-8) assay according to the instructions of the manufacturer. Briefly, 
cells were plated in 96-well plates and transfected with the corresponding miRNA with the Lipofectamine 2000. The proliferation of ovarian cancer cells was detected at the $72 \mathrm{~h}$ after transfection. $10 \mu \mathrm{L}$ of CCK-8 solution was added into the medium and incubated for additional $3 \mathrm{~h}$ at $37^{\circ} \mathrm{C}$. Absorbance of the medium at $450 \mathrm{~nm}$ was measured using the microplate reader (Bio-Rad Laboratories, Richmond, CA, USA).

\section{Western blot}

Ovarian cancer cells were transfected with miR-200a-3p mimics or scramble control miRNA using Lipofectamine 2000 for 48 h. Cells were harvested and lysed with the NP40 lysis buffer (Beyotime, Shanghai, China) on ice for $10 \mathrm{~min}$. The protein was extracted by centrifugation at $12,000 \mathrm{rpm}$ for $5 \mathrm{~min}$ at $4{ }^{\circ} \mathrm{C}$. Protein concentration was determined using the BCA assay kit (Beyotime, Shanghai, China) according to the manufacturer's instructions. Equal amount of protein was separated by SDS-PAGE and then transferred onto the nitrocellular membrane (Millipore, MA, USA). The membrane was subsequently blocked with $5 \%$ non-fat milk for $1 \mathrm{~h}$ at room temperature (RT) followed by incubated with the primary antibody overnight at $4{ }^{\circ} \mathrm{C}$. After washing twice with TBST, the membrane was incubated with HRP-conjugated secondary antibody for $1 \mathrm{~h}$ at RT. The protein bands were visualized using enhanced ECL reagents (Millipore, MA, USA). The antibodies using in this study including anti-PCDH9 (\#25090-1-AP; Proteintech, Rosemont, IL, USA) and anti-GAPDH (\#10494-1-AP; Proteintech, Rosemont, IL, USA) were purchased from the biology companies.

\section{Dual luciferase reporter assay}

The wild-type or mutated 3'-UTR of PCDH9 was amplified and inserted into the pmir-GLO-promoter vector (Promega, Madison, WI, USA). Ovarian cancer cells transfected with miR-200a-3p mimics or scramble control miRNA were co-transfected with the luciferase reporter vector containing wild-type or mutated 3'-UTR of PCDH9. After transfection for $48 \mathrm{~h}$, cells were collected and the luciferase activity was determined using the Dual Luciferase Assay Kit (Promega, Madison, WI, USA) in accordance with the manufacturer's instructions.

\section{Statistical analysis}

Data was present as mean \pm s.d. Statistical analysis was performed using the GraphPad 7.0 (GraphPad Software Inc., LaJolla, CA, USA). The difference between two groups was analyzed with the Student's $t$-test. One-way ANOVA analysis was performed to calculate the $P$-value between more than two groups. $P<0.05$ was considered as statistically significant.

\section{Results}

\section{MiR-200a-3p was up-regulated in ovarian cancer tissues and cell lines}

To explore the potential involvement of miR-200a-3p in ovarian cancer, the expression of miR-200a-3p in 50 paired ovarian cancer tissues and adjacent normal tissues was analyzed with the RT-qPCR. Compared with the normal tissues, the expression of miR-200a-3p was significantly up-regulated in ovarian cancer tissues (Figure 1A). Moreover, miR-200a-3p expression in ovarian cancer cell lines including ES2, HO8919PM, SKOV3 and HO8910 was also decreased in comparison with that of the normal ovarian surface epithelial cells HOSEpiC (Figure 1B). To better determine the clinical significance of miR-200a-3p in ovarian cancer, all the ovarian cancer patients were divided into low- and high miR-200a-3p group with the median expression level of miR-200a-3p as the cutoff value. The correlation between the expression of miR$200 a-3 p$ with the clinical characteristics was analyzed. As shown in Table 1, high level of miR-200a-3p was significantly associated with the tumor size, tumor metastasis and TNM stage. The expression level of miR-200-3p in ovarian cancer patients was included in Table S1. These results suggested that miR-200a-3p might be involved in the progression of ovarian cancer.

\section{Overexpression of miR-200a-3p promoted the growth of ovarian cancer cells}

To understand the regulation of miR-200a-3p on the growth of ovarian cancer cells, both ES2 and SKOV-3 were transfected with miR-200a-3p mimics or scramble control miRNA. The transfection efficiency was confirmed using RT-qPCR as shown in Figure 2A. The effect of miR-200a$3 p$ on the growth of ovarian cancer cells was evaluated with the CCK-8 assay, colony formation, cell cycle analysis and cell invasion. As presented in Figure 2B, overexpression of miR-200a-3p significantly promoted the proliferation of both ES2 and SKOV3 cells. The colony formation assay showed that the number of colonies formed with the ovarian cancer cells transfected with miR-200a-3p was up-regulated compared with that of the ovarian cancer expressing scramble control miRNA (Figure 2C). The promoting effect of miR$200 \mathrm{a}-3 \mathrm{p}$ on the proliferation of ovarian cancer cells was also 
A

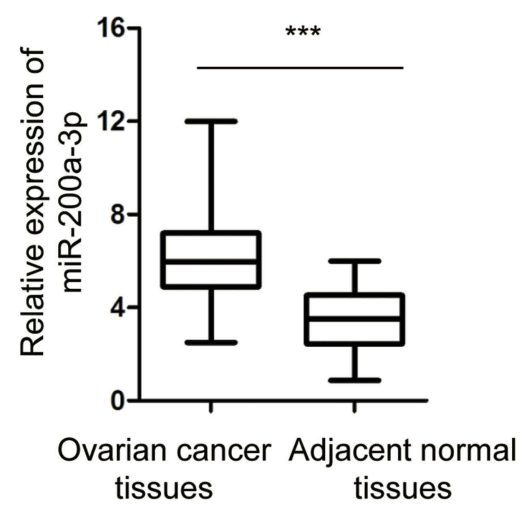

B

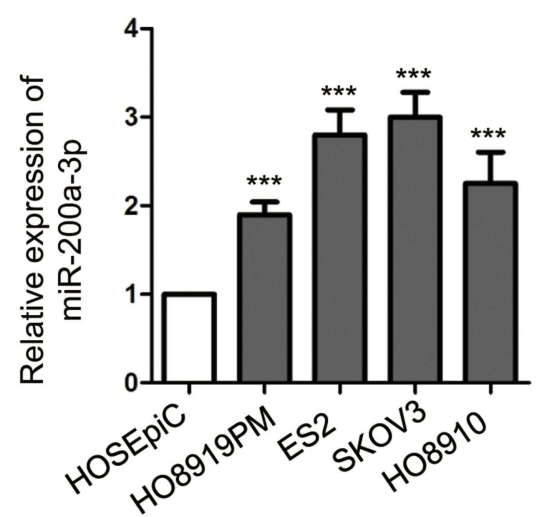

Figure I MiR-200a-3p was up-regulated in ovarian cancer. (A) RT-qPCR detection of miR-200-3p in paired ovarian cancer tissues and adjacent normal tissues. (B) The expression of miR-200a-3p in ovarian cancer cell lines and normal ovarian surface epithelial cells HOSEpiC. Data were obtained from three replicates. $* * * P<0.00 \mathrm{I}$.

Table I The correlation between the expression of miR-200a-3p and the clinical features of ovarian cancer patients

\begin{tabular}{|c|c|c|c|c|}
\hline Clinical features & No. & High-miR-200a-3p & Low-miR-200a-3p & $P$-value \\
\hline \multicolumn{5}{|l|}{ Age (years) } \\
\hline$\leq 60$ & 16 & II & 5 & $P>0.05$ \\
\hline$>60$ & 34 & 22 & 12 & \\
\hline \multicolumn{5}{|l|}{ Tumor size $(\mathrm{cm})$} \\
\hline$\leq 3$ & 20 & 8 & 12 & $P<0.001$ \\
\hline$>3$ & 30 & 25 & 5 & \\
\hline \multicolumn{5}{|c|}{ Lymph node metastasis } \\
\hline Negative & 18 & 8 & 10 & $P<0.001$ \\
\hline Positive & 32 & 25 & 7 & \\
\hline \multicolumn{5}{|l|}{ TNM stage } \\
\hline I-II & 14 & 5 & 9 & $P<0.001$ \\
\hline IIII-IV & 36 & 28 & 8 & \\
\hline
\end{tabular}

demonstrated by the finding that overexpression of miR200a-3p trigged the cell cycle progression from $\mathrm{G} 1$ to $\mathrm{S}$ phase (Figure 2D). Furthermore, as indicated in Figure 2E, up-regulated miR-200a-3p significantly promoted the invasion of both ES2 and SKOV-3 cells (Figure 2E). Collectively, these results uncovered that overexpressed miR-200a-3p promoted the growth and invasion of ovarian cancer cells.

\section{MiR-200a-3p bound the 3'-UTR of PCDH9 and negatively regulated the expression of PCDH9}

To further investigate the underlying molecular mechanism by which miR-200a-3p modulated the proliferation of ovarian cancer cells, the potential targets were predicted using the miRDB. The result showed that the $3^{\prime}$-UTR of PCDH9 contained the putative seeding sequence of miR-200a-3p (Figure 3A). The important function of PCDH9 in the development of cancers has been demonstrated by previous studies. ${ }^{36,39}$ However, the involvement of PCDH9 in ovarian cancer remains largely unknown. To validate this prediction, luciferase reporter assay was performed by transfecting both ES2 and SKOV3 with miR-200a-3p mimics/Scramble control miRNA and the wild-type/mutated PCDH9. The data showed that miR-200a-3p overexpression significantly repressed the luciferase activity of ovarian cancer cells harboring wild-type 3'-UTR of PCDH9 (Figure 3B and C). However, the inhibitory effects was almost completely abolished in ovarian cancer cells transfected with mutated 3'-UTR (Figure 3B and C). These results indicated the binding between miR-200a-3p with the 3 '-UTR of PCDH9. To confirm whether the binding affected the expression of PCDH9, both the mRNA and 
A

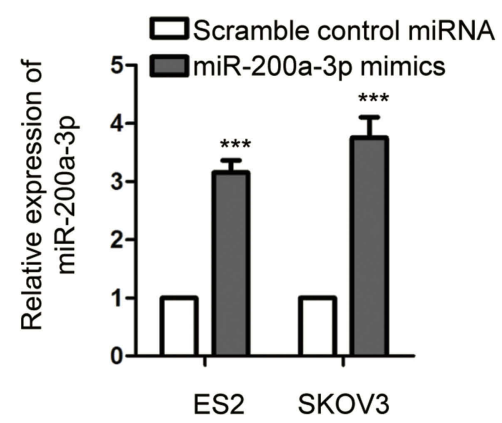

C
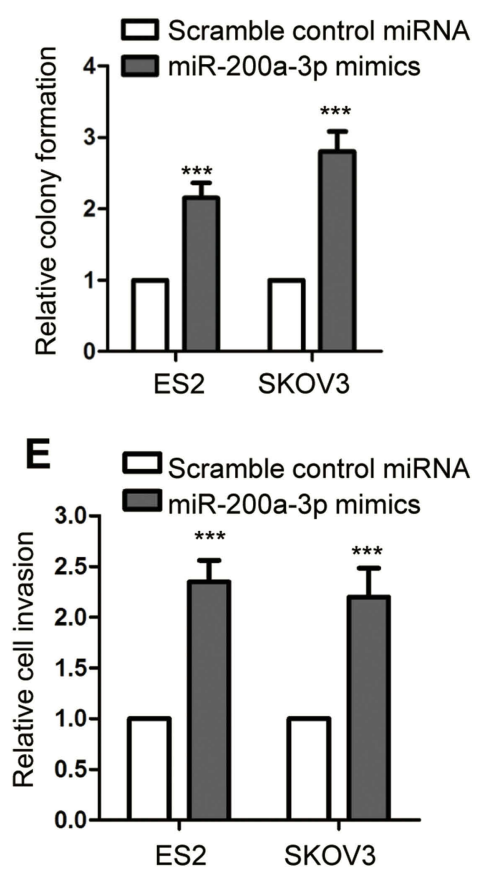

B

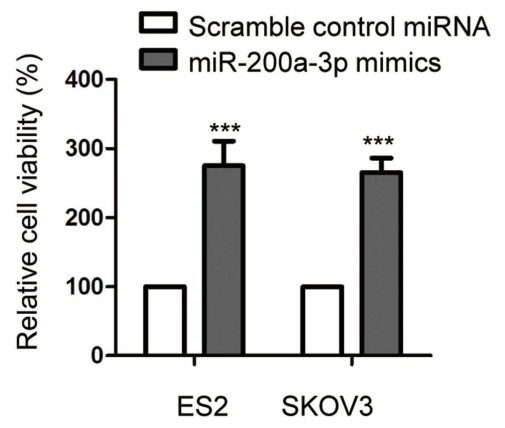

D $\square$ Scramble control miRNA

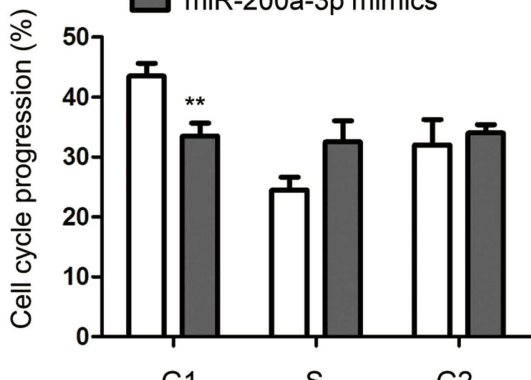

G1

G2

Figure 2 MiR-200a-3p promoted the malignant behaviors of ovarian cancer cells. (A) Confirmation of miR-200a-3p overexpression in ES2 and SKOV3 cells. Data were obtained from three replicates. (B) CCK-8 assay was performed to assess the effect of miR-200a-3p on the proliferation of both ES2 and SKOV3 cells. Data were obtained from three replicates. (C) Colony formation assay with ovarian cancer cells transfected with miR-200a-3p or control miRNA. Data were obtained from three replicates. (D) Overexpression of miR-200a-3p promoted the cell cycle progression of ES2 cells. Data were obtained from three replicates. (E) The invasion of ovarian cancer cells with overexpressed miR-200a-3p or control miRNA. Data were obtained from three replicates. $* * P<0.01, * * * p<0.001$.

protein levels of PCDH9 with the transfection of miR-200a-3p were detected. As indicated in Figure 3D, overexpression of miR-200a-3p significantly suppressed the mRNA level of PCDH9 in both ES2 and SKOV3 cells. Consistently, the protein abundance of PCDH9 was also dramatically decreased with the overexpression of miR-200a-3p in ovarian cancer cells (Figure $3 \mathrm{E}$ ). To further validate the negative modulation of miR-200a-3p on the expression of PCDH9, both ES2 and SKOV 3 cells were transfected with miR-200a-3p antagomir to knockdown the expression of miR-200a-3p (Figure 3F). As shown in Figure $3 \mathrm{G}$ and $\mathrm{H}$, both the mRNA and protein levels of PCDH9 were significantly increased with the knockdown of miR-200a-3p in ovarian cancer cells. These results suggested PCDH9 as a target of miR-200a-3p in ovarian cancer cells.

The promoting impact of mir-200a-3p on the proliferation of ovarian cancer cells was neutralized by overexpressed PCDH9

To explore whether miR-200a-3p promoted the growth of ovarian cancer cells via regulating PCDH9, both ES2 and SKOV3 cells were co-transfected with the pcDNA-FlagPCDH9 and miR-200a-3p mimics. The overexpression of PCDH9 was detected by Western blot using anti-Flag 
A

\section{Has-miR-200a-3p 5'-UAACACUGUCUGGUAACGAUGU \\ I IIIII \\ PCDH9 3'-ATTGTGACTAACTGAATGTTCACA}

B

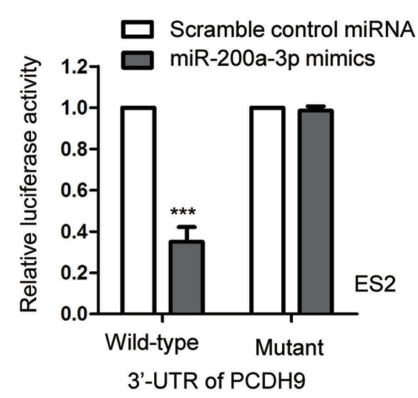

D

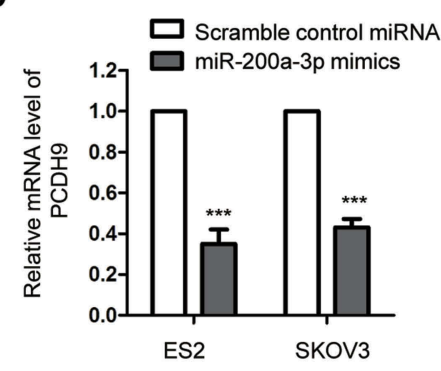

$\mathbf{F}$

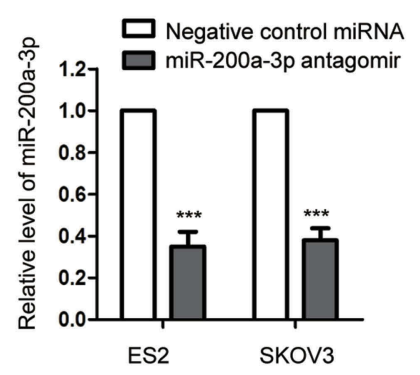

C

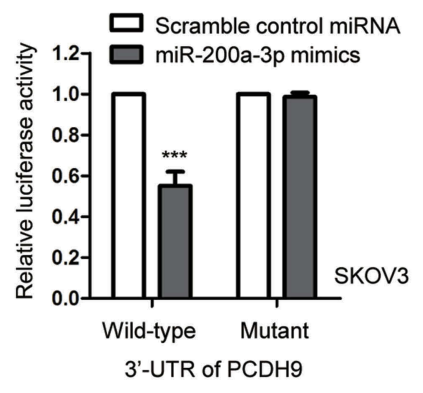

E

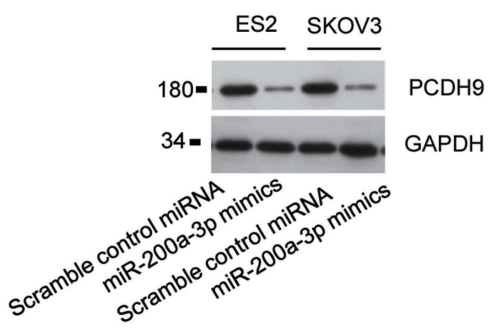

G

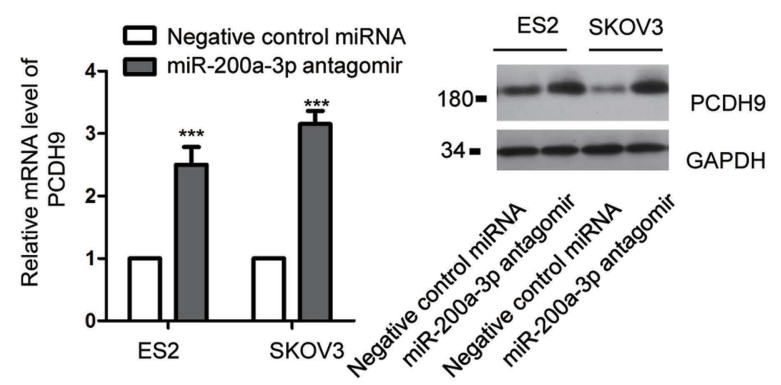

Figure $3 \mathrm{PCDH} 9$ was a target of miR-200a-3p. (A) Schematic presentation of miR-200a-3p target site in the 3 '-UTR of PCDH9. (B, C) Overexpressed miR-200a-3p decreased the luciferase activity of cells transfected with wild-type PCDH9. Data were obtained from three replicates. (D) The mRNA level of PCDH9 in ovarian cancer cells transfected with miR-200a-3p or control miRNA. Data were obtained from three replicates. (E) Western blot analysis of the PCDH9 following miR-200a-3p overexpression. Data were obtained from two replicates. (F) The confirmation of miR-200a-3p knockdown efficiency by RT-qPCR. Data were obtained from three replicates. $(\mathbf{G}, \mathbf{H})$ The mRNA and protein levels of PCDH9 in ES2 and SKOV3 cells transfected with miR-200a-3p antagomir. Data were obtained from three replicates. $* * * P<0.001$.

antibody (Figure 4A). CCK-8 assay indicated that transfection of PCDH9 significantly attenuated the promoting effect of miR-200a-3p on the proliferation of both ES2 and SKOV3 cells compared with the cells transfected with miR-200a-3p alone (Figure 4B). Consistently, the colony formation of both ES2 and SKOV3 cells was also inhibited with the co-transfection of PCDH9 compared with the control group (Figure 4C). Meanwhile, restoration of PCDH9 also attenuated the promoting effects of miR-200a-3p on the invasion of ovarian cancer cells (Figure 4D). These results suggested the critical role of PCDH9 in mediating the oncogenic function of miR-200a-3p in ovarian cancer cells.

To further support the relationship between miR-200a-3p and PCDH9, the expression of PCDH9 in ovarian cancer tissues was detected. As presented in Figure 4E, PCDH9 expression was significantly down-regulated in ovarian cancer tissues in comparison with that of the corresponding normal tissues. The decreased level of PCDH9 was also 
A

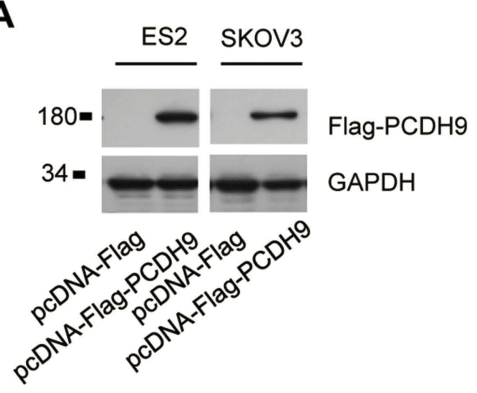

C

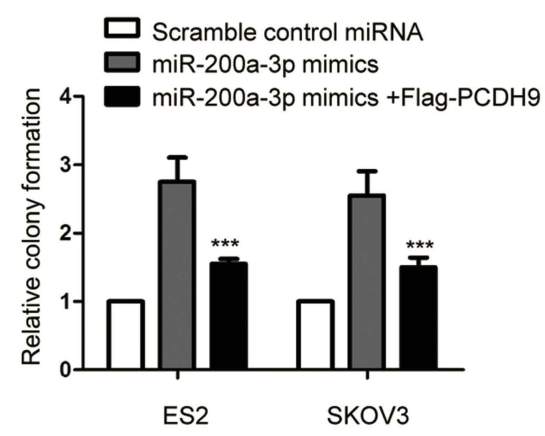

E

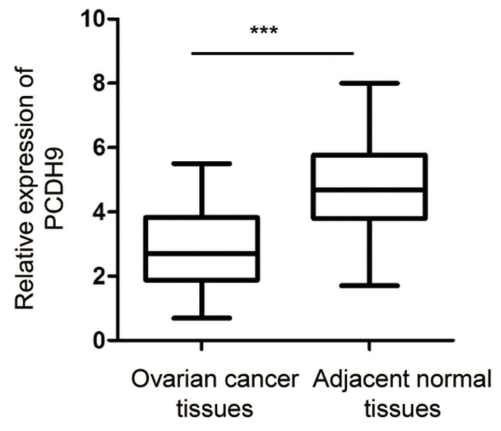

G

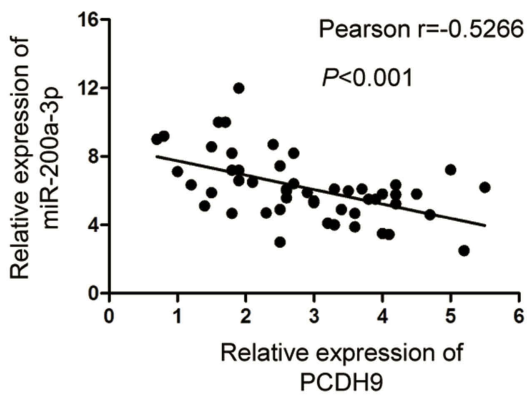

B

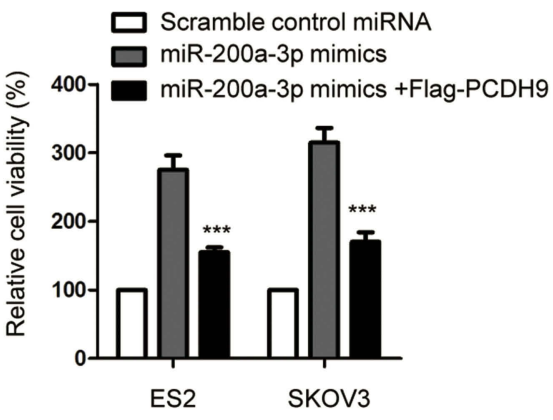

D

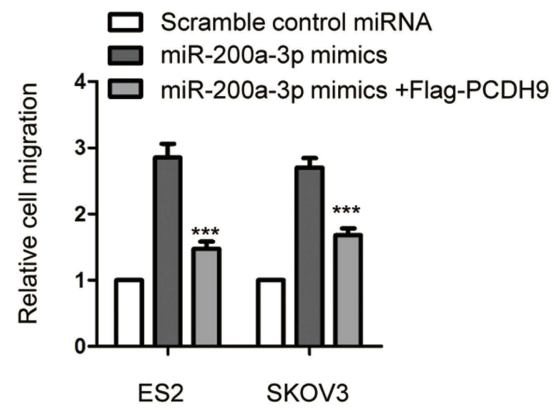

F

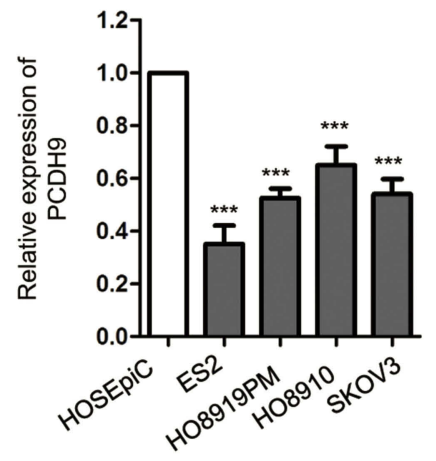

Figure 4 PCDH9 overexpression attenuated the promoting effect of miR-200a-3p on the proliferation of ovarian cancer cells. (A) Western blot analysis of overexpressed Flag-PCDH9 in both ES2 and SKOV3 cells. Data were obtained from two replicates. (B) CCK-8 assay to evaluate the effect of PCDH9 overexpression on miR-200a-3p induced cell growth. Data were obtained from three replicates. (C) Overexpression of PCDH9 inhibited the colon formation induced by miR-200a-3p. Data were obtained from three replicates. (D) Restoration of PCDH9 suppressed the invasion of ovarianc cancer cells induced by miR-200a-3p. (E) RT-qPCR detection of PCDH9 expression in paired ovarian cancer tissues and adjacent normal tissues. Data were obtained from three replicates. (F) The expression of PCDH9 in normal cell HOSEpiC and ovarian cancer cell lines. Data were obtained from three replicates. (G) Spearman test was performed to analyze the correlation between the expression of miR-200a-3p and PCDH9. $* * * P<0.001$

observed in a serial of ovarian cancer cells compared with the normal cells (Figure 4F). To characterize the correlation between the expressions of miR-200a-3p and PCDH9, spearman test was performed. The analysis indicated the significant negative correlation between the level of miR200a-3p and PCDH9 (Figure 4G). These results further supported the negative regulation of miR-200a-3p on the expression of PCDH9 in ovarian cancer cells. 


\section{Discussion}

An increasing boy of evidence has indicated the important function of miRNAs in ovarian cancer. MiR-200a-3p was overexpressed and played oncogenic roles in a variety of cancers. $^{17,18}$ Recent study showed that miR-200a-3p increased the 5-fluorouracil resistance of hepatocellular carcinoma cells by regulating the dual specificity phosphatase 6 expression. ${ }^{17}$ The oncogenic role of miR-200a-3p was also established in esophageal cancer by post-transcriptionally modulating the expression of cytoplasmic collapsing response mediator protein-1. ${ }^{18}$ MiR-200a-3p promoted the cell survival after 5-fluorouracil treatment in human hepatocellular carcinoma. ${ }^{17}$ Interestingly, it was also reported that miR-200a-3p regulated the expression of SPAG9 and inhibited the proliferation of renal cell carcinoma. $^{40}$ Recent study also demonstrated that miR200 family members suppressed the epithelial-mesenchymal transition of lung cancer. ${ }^{41}$ The tumor suppressive role of miR-200 family is closely associated with the glinoma initiation, progression and metastasis. ${ }^{42}$ These findings suggested the opposite function of miR-200a-3p and other miR-200 family members in cancers. In the present study, we found that miR-200a-3p was significantly upregulated in ovarian cancer tissues and cell lines. Overexpressed miR-200a-3p was remarkably associated with the advanced progression of ovarian cancer patients. These results suggested that miR-200a-3p was possibly involved in the pathogenesis of ovarian cancer and was a promising biomarker for the prognosis of ovarian cancer.

The potential oncogenic role of miR-200a-3p in ovarian cancer was further evaluated by in vitro functional experiments. Overexpression of miR-200a-3p significantly promoted the proliferation and colony formation of ovarian cancer cells. Moreover, highly expressed miR-200a-3p enhanced the cell cycle progression and the invasion of ovarian cancer cells. Considering the promoting impact of miR-200a-3p in regulating the malignant behaviors of ovarian cancer cells, it might be necessary to determine the effect of miR-200a-3p on the resistance of ovarian cancer cells to chemotherapy and radiotherapy. Additionally, the potential oncogenic role of miR-200a-3p in ovarian cancer calls for further in vivo validation.

PCDH9 is a member of the protocadherin family and cadherin superfamily. ${ }^{37}$ Recent studies identified PCDH9 as a potential tumor suppressor in a variety of cancers. Down-regulation of PCDH9 was associated with the poor prognosis and worse survival rate of cancer patients. ${ }^{36}$ It is well established that aberrant miRNA profiles regulated the malignant phenotypes through regulating the expression of target genes. PCDH9 was identified as the downstream target of miR-215-5p in gliomas and inhibited the growth of glioma cells. ${ }^{35}$ In this study, the possible binding sites of miR-200a-3p at the 3'-UTR of PCDH9 was predicted with the miRDB. Functional analysis showed that miR-200a-3p bound the 3'-UTR of PCDH9 and suppressed the expression of PCDH9 in ovarian cancer cells. Significant inversed correlation between the expression of miR-200a-3p and PCDH9 was observed in ovarian cancer tissues. Restored PCDH9 significantly reversed the promoting effect of miR-200a-3p on the proliferation of ovarian cancer cells. These results demonstrated the essential role of PCDH9 in mediating the potential oncogenic function of miR-200a-3p in ovarian cancer. As miRNAs have multiple targets, the involvement of other targets in addition to PCDH9 requires further investigation.

In conclusions, our findings demonstrated that miR200a-3p was up-regulated in ovarian cancer and significantly associated with the advanced progression of ovarian cancer patients. Overexpressed miR-200a-3p promoted the proliferation of ovarian cancer cells via targeting PCDH9. Our results provided the novel functional mechanism of miR-200a-3p/PCDH9 axis in ovarian cancer. Targeting miR-200a-3p might be a promising strategy for the treatment of ovarian cancer.

\section{Acknowledgment}

This work was supported by the Huai'an Science and Technology Development Fund (HAB201725).

\section{Disclosure}

The authors report no conflicts of interest in this work.

\section{References}

1. Amos CI, Struewing JP. Genetic epidemiology of epithelial ovarian cancer. Cancer. 1993;71(2 Suppl):566-572. doi:10.1002/cncr.282071 0212

2. Haruta S, Furukawa N, Yoshizawa Y, et al. Molecular genetics and epidemiology of epithelial ovarian cancer (Review). Oncol Rep. 2011;26(6):1347-1356. doi:10.3892/or.2011.1456

3. Webb PM, Jordan SJ. Epidemiology of epithelial ovarian cancer. Best Pract Res Clin Obstet Gynaecol. 2017;41:3-14. doi:10.1016/j. bpobgyn.2016.08.006

4. Xie HH, Huan WT, Han JQ, Ren WR, Yang LH. MicroRNA-663 facilitates the growth, migration and invasion of ovarian cancer cell by inhibiting TUSC2. Biol Res. 2019;52(1):18.

5. Wu KL, Tsai YM, Lien CT, Kuo PL, Hung AJ. The roles of MicroRNA in lung cancer. Int J Mol Sci. 2019;20(7):1611.

6. Mohr AM, Mott JL. Overview of microRNA biology. Semin Liver Dis. 2015;35(1):3-11. doi:10.1055/s-0034-1397344 
7. Bartel DP. MicroRNAs: genomics, biogenesis, mechanism, and function. Cell. 2004;116(2):281-297.

8. Fabian MR, Sonenberg N, Filipowicz W. Regulation of mRNA translation and stability by microRNAs. Annu Rev Biochem. 2010;79:351-379. doi:10.1146/annurev-biochem-060308-103103

9. Krol J, Loedige I, Filipowicz W. The widespread regulation of microRNA biogenesis, function and decay. Nat Rev Genet. 2010;11 (9):597-610. doi:10.1038/nrg2843

10. Deb B, Uddin A, Chakraborty S. miRNAs and ovarian cancer: an overview. J Cell Physiol. 2018;233(5):3846-3854. doi:10.1002/jcp. 26095

11. Nakamura K, Sawada K, Yoshimura A, Kinose Y, Nakatsuka E, Kimura T. Clinical relevance of circulating cell-free microRNAs in ovarian cancer. Mol Cancer. 2016;15(1):48. doi:10.1186/s12943-0160536-0

12. Mandilaras V, Vernon M, Meryet-Figuiere M, et al. Updates and current challenges in microRNA research for personalized medicine in ovarian cancer. Expert Opin Biol Ther. 2017;17(8):927-943. doi:10.1080/14712598.2017.1340935

13. Zhou J, Zhang C, Zhou B, Jiang D. miR-183 modulated cell proliferation and apoptosis in ovarian cancer through the TGF-beta/Smad4 signaling pathway. Int J Mol Med. 2019;43(4):1734-1746. doi:10.3892/ijmm.2019.4082

14. Xing F, Wang S, Zhou J. The expression of MicroRNA-598 inhibits ovarian cancer cell proliferation and metastasis by targeting URI. Mol Ther Oncolytics. 2019;12:9-15. doi:10.1016/j.omto.2018.12.002

15. Xiao S, Li Y, Pan Q, et al. MiR-34c/SOX9 axis regulates the chemoresistance of ovarian cancer cell to cisplatin-based chemotherapy. J Cell Biochem. 2019;120(3):2940-2953. doi:10.1002/jcb.26865

16. Shi T, Hua Q, Ma Z, Lv Q. Downregulation of miR-200a-3p induced by hepatitis B Virus X ( $\mathrm{HBx})$ Protein promotes cell proliferation and invasion in HBV-infection-associated hepatocarcinoma. Pathol Res Pract. 2017;213(12):1464-1469. doi:10.1016/j.prp.2017.10.020

17. Lee H, Kim C, Kang H, et al. microRNA-200a-3p increases 5fluorouracil resistance by regulating dual specificity phosphatase 6 expression. Exp Mol Med. 2017;49(5):e327. doi:10.1038/emm.2017. 33

18. Zang Y, Tai Y, Wan B, Jia X. miR-200a-3p promotes the proliferation of human esophageal cancer cells by post-transcriptionally regulating cytoplasmic collapsin response mediator protein-1. Int $\mathrm{J} \mathrm{Mol} \mathrm{Med}$. 2016;38(5):1558-1564. doi:10.3892/ijmm.2016.2758

19. Ashaie MA, Chowdhury EH. Cadherins: the superfamily critically involved in breast cancer. Curr Pharm Des. 2016;22(5):616-638. doi:10.2174/138161282205160127095338

20. van Roy F. Beyond E-cadherin: roles of other cadherin superfamily members in cancer. Nat Rev Cancer. 2014;14(2):121-134. doi:10.10 $38 / \operatorname{nrc} 3647$

21. Sui X, Wang D, Geng S, Zhou G, He C, Hu X. Methylated promoters of genes encoding protocadherins as a new cancer biomarker family. Mol Biol Rep. 2012;39(2):1105-1111. doi:10.1007/s11033-011-08378

22. El Hajj N, Dittrich M, Haaf T. Epigenetic dysregulation of protocadherins in human disease. Semin Cell Dev Biol. 2017;69:172-182. doi:10.1016/j.semcdb.2017.07.007

23. Yu JS, Koujak S, Nagase S, et al. PCDH8, the human homolog of PAPC, is a candidate tumor suppressor of breast cancer. Oncogene. 2008;27(34):4657-4665. doi:10.1038/onc.2008.101

24. Lin YL, Ma JH, Luo XL, Guan TY, Li ZG. Clinical significance of protocadherin-8 (PCDH8) promoter methylation in bladder cancer. $J$ Int Med Res. 2013;41(1):48-54. doi:10.1177/0300060513475571

25. Lin YL, Wang YL, Ma JG, Li WP. Clinical significance of protocadherin 8 (PCDH8) promoter methylation in non-muscle invasive bladder cancer. J Exp Clin Cancer Res. 2014;33:68.
26. Zhang C, Peng Y, Yang F, Qin R, Liu W, Zhang C. PCDH8 is frequently inactivated by promoter hypermethylation in liver cancer: diagnostic and clinical significance. J Cancer. 2016;7(4):446-452. doi:10.7150/jca.13065

27. Lin YL, Li YL, Ma JG. Aberrant promoter methylation of protocadherin8 (PCDH8) in serum is a potential prognostic marker for low gleason score prostate cancer. Med Sci Monit. 2017;23:4895-4900. doi:10.12659/MSM.904366

28. Li Z, Li W, Xie J, et al. Epigenetic inactivation of PCDH10 in human prostate cancer cell lines. Cell Biol Int. 2011;35(7):671-676.

29. Li Z, Chim JC, Yang M, Ye J, Wong BC, Qiao L. Role of PCDH10 and its hypermethylation in human gastric cancer. Biochim Biophys Acta. 2012;1823(2):298-305. doi:10.1016/j.bbamcr.2011.11.011

30. Zhong X, Zhu Y, Mao J, Zhang J, Zheng S. Frequent epigenetic silencing of PCDH10 by methylation in human colorectal cancer. $J$ Cancer Res Clin Oncol. 2013;139(3):485-490. doi:10.1007/s00432012-1353-5

31. Yang Y, Liu J, Li X, Li JC. PCDH17 gene promoter demethylation and cell cycle arrest by genistein in gastric cancer. Histol Histopathol. 2012;27(2):217-224. doi:10.14670/HH-27.217

32. Lin YL, Deng QK, Wang YH, Fu XL, Ma JG, Li WP. Aberrant Protocadherin17 (PCDH17) methylation in serum is a potential predictor for recurrence of early-stage prostate cancer patients after radical prostatectomy. Med Sci Monit. 2015;21:3955-4690. doi:10.12659/msm.896763

33. Wang Y, Yu Y, Ye R, et al. An epigenetic biomarker combination of PCDH17 and POU4F2 detects bladder cancer accurately by methylation analyses of urine sediment DNA in Han Chinese. Oncotarget. 2016;7(3):2754-2764. doi:10.18632/oncotarget.6666

34. Wu Y, Jiang G, Zhang N, et al. HOXA9, PCDH17, POU4F2, and ONECUT2 as a urinary biomarker combination for the detection of bladder cancer in Chinese patients with hematuria. Eur Urol Focus. 2018. doi:10.1016/j.euf.2018.09.016

35. Wang C, Chen Q, Li S, et al. Dual inhibition of PCDH9 expression by miR-215-5p up-regulation in gliomas. Oncotarget. 2017;8 (6):10287-10297. doi:10.18632/oncotarget.14396

36. Chen Y, Xiang H, Zhang Y, Wang J, Yu G. Loss of PCDH9 is associated with the differentiation of tumor cells and metastasis and predicts poor survival in gastric cancer. Clin Exp Metastasis. 2015;32 (5):417-428. doi:10.1007/s10585-015-9712-7

37. Lv J, Zhu P, Zhang X, et al. PCDH9 acts as a tumor suppressor inducing tumor cell arrest at G0/G1 phase and is frequently methylated in hepatocellular carcinoma. Mol Med Rep. 2017;16(4):44754482. doi:10.3892/mmr.2017.7193

38. Xie Z, Zhou F, Yang Y, et al. Lnc-PCDH9-13:1 is a hypersensitive and specific biomarker for early hepatocellular carcinoma. EBioMedicine. 2018;33:57-67. doi:10.1016/j.ebiom.2018.06.026

39. Izycka N, Sterzynska K, Januchowski R, Nowak-Markwitz E. Semaphorin 3A (SEMA3A), protocadherin 9 (PCdh9), and S100 calcium binding protein A3 (S100A3) as potential biomarkers of carcinogenesis and chemoresistance of different neoplasms, including ovarian cancer - review of literature. Ginekol Pol. 2019;90(4):223227. doi:10.5603/GP.2019.0040

40. Wang X, Jiang F, Song H, Li X, Xian J, Gu X. MicroRNA-200a-3p suppresses tumor proliferation and induces apoptosis by targeting SPAG9 in renal cell carcinoma. Biochem Biophys Res Commun. 2016;470(3):620-626. doi:10.1016/j.bbrc.2016.01.095

41. Liu C, Hu W, Li LL, et al. Roles of miR-200 family members in lung cancer: more than tumor suppressors. Future Oncol. 2018;14 (27):2875-2886. doi:10.2217/fon-2018-0155

42. Peng L, Fu J, Ming Y. The miR-200 family: multiple effects on gliomas. Cancer Manag Res. 2018;10:1987-1992. doi:10.2147/CMAR.S160945 


\section{Publish your work in this journal}

OncoTargets and Therapy is an international, peer-reviewed, open access journal focusing on the pathological basis of all cancers, potential targets for therapy and treatment protocols employed to improve the management of cancer patients. The journal also focuses on the impact of management programs and new therapeutic agents and protocols on patient perspectives such as quality of life, adherence and satisfaction. The manuscript management system is completely online and includes a very quick and fair peer-review system, which is all easy to use. Visit http://www.dovepress.com/ testimonials.php to read real quotes from published authors. 